\title{
Engineering quantum anomalous Hall phases with orbital and spin degrees of freedom
}

\author{
Hongbin Zhang, ${ }^{*}$ Frank Freimuth, Gustav Bihlmayer, Marjana Ležaić, Stefan Blügel, and Yuriy Mokrousov ${ }^{\dagger}$ \\ Peter Grünberg Institut and Institute for Advanced Simulation, Forschungszentrum Jülich and JARA, D-52425 Jülich, Germany
}

(Received 21 January 2013; revised manuscript received 8 May 2013; published 23 May 2013)

\begin{abstract}
Combining tight-binding models and first-principles calculations, we investigate the quantum anomalous Hall (QAH) effect induced by intrinsic spin-orbit coupling (SOC) in buckled honeycomb lattice with $s p$ orbitals in an external exchange field. Detailed analysis reveals that nontrivial topological properties can arise utilizing not only spin but also orbital degrees of freedom in the strong SOC limit, when the bands acquire nonzero Chern numbers upon undergoing the so-called orbital purification. As a prototype of a buckled honeycomb lattice with strong SOC we choose the $\mathrm{Bi}(111)$ bilayer, analyzing its topological properties in detail. In particular, we show the emergence of several QAH phases upon spin exchange of the Chern numbers as a function of SOC strength and magnitude of the exchange field. Interestingly, we observe that in one of such phases, namely, in the quantum spin Chern insulator phase, the quantized charge and spin Hall conductivities coexist. We consider the possibility of tuning the SOC strength in the Bi bilayer via alloying with isoelectronic $\mathrm{Sb}$, and speculate that exotic properties could be expected in such an alloyed system owing to the competition of the topological properties of its constituents. Finally, we demonstrate that $3 d$ dopants can be used to induce a sizable exchange field in the $\mathrm{Bi}(111)$ bilayer, resulting in nontrivial Chern insulator properties.
\end{abstract}

DOI: 10.1103/PhysRevB.87.205132

PACS number(s): 75.70.-i, 73.20.At, 75.30.Kz

\section{INTRODUCTION}

In recent years, dissipationless charge and spin transport phenomena have drawn very intensive attention. After the topological nature of the anomalous Hall effect (AHE) in ferromagnets was discovered and understood, ${ }^{1}$ the existence of the spin Hall $^{2,3}$ and quantum spin Hall (QSH) effects ${ }^{4,5}$ was theoretically proposed and experimentally demonstrated. Nevertheless, the experimental observation of the so-called quantum anomalous Hall effect (QAHE), ${ }^{6}$ characterized by a quantization of the anomalous Hall conductivity, is still missing, despite various theoretical proposals ${ }^{7-11}$ and recent experimental advances in this direction. ${ }^{12}$

Numerous studies on the QSH effect have spawned a fascinating field focusing on the topologically nontrivial states of matter, in particular, topological insulators (TIs). ${ }^{13,14}$ Remarkable physical properties of TIs originate from the gapless surface states, guaranteed by a finite bulk gap and time-reversal symmetry. It is plausible to seek QAH effects by introducing time-reversal broken perturbations to TIs while keeping the topological nontriviality, e.g., via a proximity effect. $^{14}$ Among all known TIs, ${ }^{15}$ two-dimensional (2D) systems are of particular interest, such as graphene, ${ }^{4} \mathrm{HgTe}$ quantum wells, ${ }^{5} \mathrm{AlSb} / \mathrm{InAs} / \mathrm{GaSb}$ quantum wells, ${ }^{16}$ silicene, ${ }^{17}$ and $\mathrm{Bi}(111)$ bilayers (BLs). ${ }^{18,19}$ In the past, a lot of work has been done based on the extended Kane-Mele model ${ }^{4}$ in the context of graphene. ${ }^{9,20-24}$ In these studies, only the $\sigma$ band of the $p_{z}$ orbitals is considered, with key terms to induce topological nontriviality being effective projected spin-orbit and Rashba spin-orbit interactions, arising from second-order perturbative processes. ${ }^{25}$ The strength of intrinsic atomic spin-orbit coupling of carbon is limited, although it can be enhanced in fine-tuned systems. ${ }^{26}$

In the quantized case of the AHE in two dimensions, the value of the anomalous Hall conductivity (AHC) is proportional to the so-called (first) Chern number, obtained as an integral of the Berry curvature of the occupied states in the Brillouin zone. This integer Chern number, which characterizes the "phase" complexity of the manifold of Bloch electrons as a whole, has its roots in solid state physics since the discovery of the quantum Hall effect and its theoretical understanding by Thouless et al. in $1982 .{ }^{27}$ Shortly after that, Haldane suggested a spinless model to realize the QAHE without an external magnetic field, ${ }^{6}$ an effort, which conceptually led to the theoretical suggestion of graphene as a first topological insulator. ${ }^{4}$ In the latter case, the Chern numbers of spin-up and -down electrons are nonzero due to a microscopic realization of the Haldane's model, but they are opposite to each other. The generality of this counteracting nature of the Chern numbers in TIs leads eventually to the concept of the spin Chern numbers, which can be used to characterize the TI phases in time-reversal broken situations. ${ }^{28}$ Ultimately, the realization of the QAHE is hinged on the presence of points in the electronic structure, at which otherwise separate spin bands interact with each other, and exchange corresponding spin Chern numbers, to give a global nonvanishing Chern number in the system. The mechanism behind such a Chern number exchange is strongly dependent on the details of symmetry, hybridization, and microscopic structure of the spin-orbit interaction in the system, making search for systems which could exhibit nonzero QAHE difficult, yet definitely rewarding since the physics involved is possibly very rich.

Among other materials, bismuth (111) bilayer has been shown to host nontrivial topological properties due to the strong SOC of $\mathrm{Bi}^{18,19,29}$ In our previous work, we used the concept of the spin Chern number to characterize the topological phase transitions in $\mathrm{Bi}(111)$ bilayers with respect to the strength of an external exchange field. ${ }^{29}$ Here, we extend this work further, and demonstrate the microscopic origin of the QAH phases found in $\mathrm{Bi}(111)$ bilayers when utilizing both orbital and spin degrees of freedom. Based on a general tight-binding model for buckled honeycomb lattices with $s p$ orbitals, we illustrate different Haldane-inspired ${ }^{6}$ mechanisms which can lead to a nonzero Chern number in 
each individual band upon employing the spin and orbital complexity (Sec. II). We find that in addition to $p_{z}$ orbital based physics, in the strong SOC limit, orbital angular momentum purification can also lead to nontrivial topological properties. We further clarify the ways behind achieving the QAHE at the so-called spin-mixing points in the electronic structure. Finally, using first-principles techniques, we show that various topological phases occur in a realization of a buckled $s p$ honeycomb model [the $\mathrm{Bi}(111)$ bilayer] with respect to the SOC strength and magnitude of an external exchange field (Sec. III). In particular, we suggest the existence of a so-called quantum spin Chern insulator phase, which is characterized by the quantization of both the transverse charge and spin conductivities. We conclude by discussing how to realize the tuning of SOC strength by alloying $\mathrm{Bi}$ with $\mathrm{Sb}$, and the effect of an exchange field by introducing magnetic substrates and/or dopants.

\section{MODEL ANALYSIS}

To illustrate how nonzero Chern numbers and nontrivial QAH phases can arise, we first consider a generic tight-binding Hamiltonian of $s p$ orbitals on a buckled honeycomb lattice in the $x-y$ plane:

$$
H=\sum_{i, j} t_{i, j} c_{i}^{\dagger} c_{j}+\sum_{i} c_{i}^{\dagger}\left(\epsilon_{i} \mathbb{I}+B s_{z}\right) c_{i}+H_{\mathrm{SOC}},
$$

where the first term presents the kinetic hopping with $t_{i, j}$ $\left(i, j=s, p_{x}, p_{y}, p_{z}\right)$ as the hopping parameters. The second term reflects an orbital-dependent onsite energy $\epsilon_{i}$ and the interaction with the Zeeman exchange field $B$ directed along the $z$ axis, with $\mathbb{I}\left(s_{z}\right)$ as the identity (Pauli) matrix. The third term in Hamiltonian (1) is the onsite SOC Hamiltonian. In order to facilitate the analysis, $s, p_{z}$, and $\left\{p_{x}, p_{y}\right\}$ bands are artificially separated from each other by imposing a rigid shift of $\epsilon_{i}$. To identify different origins of the QAHE, the spin-orbit interaction is further decomposed into spin-conserving and spin-flip parts:

$$
H_{\mathrm{SOC}}=\xi \mathbf{l} \cdot \mathbf{s}=\xi l_{z} s_{z}+\xi\left(l^{+} s^{-}+l^{-} s^{+}\right) / 2,
$$

where $\mathbf{l}$ (s) is the orbital (spin) angular momentum operator, and $\xi$ is the electron-shell averaged atomic SOC strength. Since in this work we choose the direction of the spin polarization to be aligned along the $z$ direction, the spin-conserving part of the SOC Hamiltonian $\xi l_{z} s_{z}$ couples $\left\{p_{x}, p_{y}\right\}$ orbitals, while the spin-flip part of Eq. (2), $\xi\left(l^{+} s^{-}+l^{-} s^{+}\right) / 2$, couples $p_{z}$ and $\left\{p_{x}, p_{y}\right\}$ orbitals via a flip of spin and \pm 1 change in the orbital quantum number. Further, we take the Slater-Koster tight-binding parameters of $\mathrm{Bi}$, provided in Ref. 30 for the values of hopping integrals of the model $t_{i, j}$.

Before proceeding further with the analysis of Eq. (1), we would like to remind the reader of the Haldane model, ${ }^{6}$ which is a generic model exhibiting an emergence of the QAH effect on a honeycomb lattice without an external magnetic field. The key mechanism in this model is the complex hopping between the next-nearest-neighbor (NNN) orbitals, which leads to a sublattice-dependent "magnetic field" with zero flux through the overall hexagonal plaquette. Therefore, for an individual band, a nonzero Chern number can be induced due to the complex NNN hoppings, as demonstrated in Ref. 6. There are various interactions which can induce complex hopping integrals, and we considered onsite atomic SOC in this work. Since the matrix elements of SOC are complex conjugate for spin-up and -down electrons, the phases of the complex hoppings are of opposite sign for spin-up and -down electrons and the same holds for the Chern numbers. In the following sections, we will demonstrate how such complex hoppings can be engineered for bands with different orbital characters, leading to topological insulator phases when the time-reversal symmetry is not broken. Furthermore, QAHE phases are shown to occur when time-reversal breaking exchange fields are introduced.

\section{A. $p_{z}$ orbitals}

First, we consider the case of the $p_{z}$ bands, particularly relevant in graphene physics, well separated from $\left\{p_{x}, p_{y}\right\}$ and $s$ states. Generally, there are two ways to induce nontrivial Chern numbers in the $p_{z}$ bands via generating an effective next-nearest-neighbor complex hopping due to intrinsic atomic SOC. First, on a buckled honeycomb lattice, $p_{z}$ orbitals can hybridize directly with the $\left\{p_{x}, p_{y}\right\}$ orbitals, and complex hoppings can be induced via the spin-conserving part of SOC which acts between $p_{x}$ and $p_{y}$ states. As illustrated with a sketch in Fig. 1(a), in this mechanism the corresponding virtual transitions read as ${ }^{31}$

$$
\left|p_{z}^{\mathrm{A} \uparrow}\right\rangle \stackrel{t_{\mathrm{NN}}}{\rightarrow}\left|p_{x, y}^{\mathrm{B}} \uparrow\right\rangle \stackrel{\xi l_{z} s_{z}}{\rightarrow}\left|p_{x, y}^{\mathrm{B}} \uparrow\right\rangle \stackrel{t_{\mathrm{NN}}}{\rightarrow}\left|p_{z}^{\mathrm{A} \uparrow}\right\rangle,
$$

where $t_{\mathrm{NN}}$ indicates the direct hopping between $p_{z}$ and $p_{x, y}$ orbitals on the neighboring sites, while superscripts A and $\mathrm{B}$ denote the nearest-neighbor atomic sites in sublattices $\mathrm{A}$ and $\mathrm{B}$.

For the resulting NNN hopping, we obtain $t_{\mathrm{NNN}} \sim \xi$. Importantly, the effective hoppings are opposite in sign for $p_{z}$ orbitals of a given spin on the $\mathrm{A}$ and $\mathrm{B}$ sublattices, hence giving rise to a finite gap $\Delta_{1} \sim 2 \xi$ at the $\mathrm{K}\left(\mathrm{K}^{\prime}\right)$ high-symmetry points in the Brillouin zone, as shown in Fig. 1(a). In this figure, a small exchange field has been applied in order to induce a splitting between spin-up and -down bands for visibility. Since in the presence of spin-conserving SOC the spin channels are not coupled to each other, we can define the Chern numbers for spin-up and -down bands separately (shown with numbers next to the bands in Fig. 1). Without an exchange field, the spin-up and -down bands would be degenerate and the $\Delta_{1}$ gap would be positioned at the Fermi energy. This gap is topologically nontrivial, in direct accordance to Haldane's arguments, ${ }^{6}$ and nonzero Chern number of the occupied spin-up band is opposite to that of the spin-down band [Fig. 1(a)]. This, without an exchange field, would result in suppression of the QAH effect and emergence of the QSH state (see Table I).

Second, onsite spin-flip SOC can give rise to complex next-nearest-neighbor hopping too, even if there is no direct hybridization between $p_{z}$ and $\left\{p_{x}, p_{y}\right\}$ orbitals [Fig. 1(b)]. In this case, the corresponding virtual transitions are ar, $^{25,32}$

$$
\left.\left|p_{z}^{\mathrm{A} \uparrow}\right\rangle \stackrel{\xi_{\text {flip }}}{\rightarrow}\left|p_{x, y}^{\mathrm{A}} \downarrow\right\rangle \stackrel{t_{\mathrm{NN}}}{\rightarrow}\left|p_{x, y}^{\mathrm{B}} \downarrow\right\rangle \stackrel{t_{\mathrm{NN}}}{\rightarrow} \mid p_{x, y}^{\mathrm{A}} \downarrow\right) \stackrel{\xi_{\text {flip }}}{\rightarrow}\left|p_{z}^{\mathrm{A}} \uparrow\right\rangle,
$$



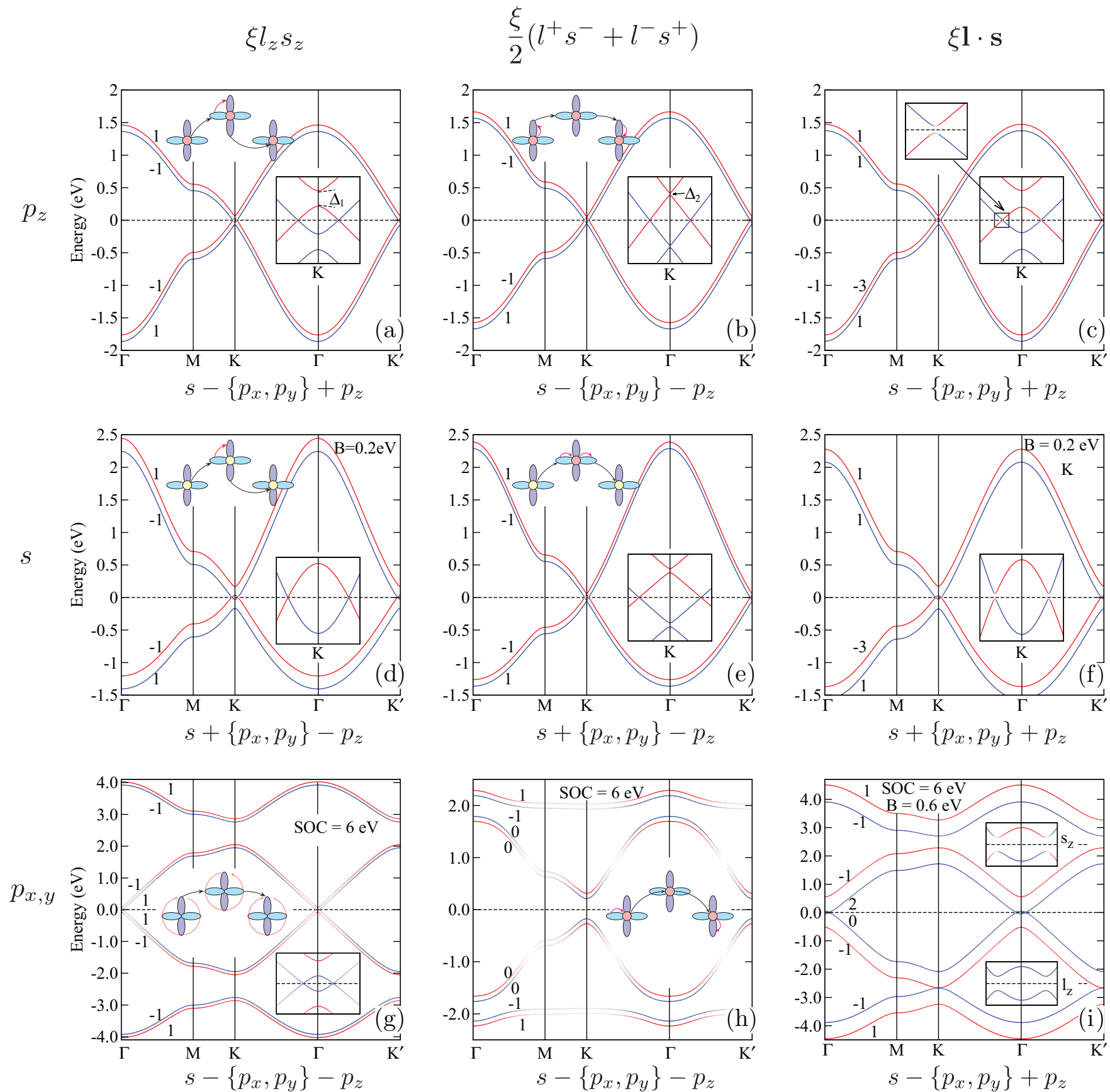

FIG. 1. (Color online) Topological analysis of $p_{z}[(\mathrm{a})-(\mathrm{c})], s[(\mathrm{~d})-(\mathrm{f})]$, and $\left\{p_{x}, p_{y}\right\}[(\mathrm{g})-(\mathrm{i})]$ bands in a buckled honeycomb bilayer. The electronic structure is obtained using the tight-binding parameters for bulk Bi as listed in Ref. 30, with an additional artificial rigid shift of onsite energies yielding separation of $s, p_{z},\left\{p_{x}, p_{y}\right\}$ states in energy. Only nearest-neighbor (NN) hoppings are considered, with $\mathrm{V}_{p p \pi}=0$ (this does not affect our conclusions, see the main text). A small exchange field has been applied in all cases, yielding a QAH state in (c), (f), and (i). Without an exchange field, systems in (a), (b), (d), (e), (g), and (h) would be in a TI state (see also Table I). Without specification, the spin-orbit coupling parameter $\xi=4.5 \mathrm{eV}$ and exchange field $B=0.1 \mathrm{eV}$ are used. Left column [(a), (d), (g)] [middle column (b), (e), (h)] corresponds to the case with only spin-conserving SOC (spin-flip SOC) included, while the full SOC is considered in the right column [(c), (f), (i)]. Red (blue) in (a)-(f) and upper inset of (i) stand for the spin-down (spin-up) states, while in (g)-(i) and lower inset of (i) for the states with orbital magnetic number $m=+1(m=-1)$. Dashed horizontal lines indicate the Fermi energy. In the case of isolated bands, numbers denote the Chern number for each individual band, while for overlapping bands, numbers stand for the Chern numbers of nonhybridizing spin-up (red) and spin-down (blue) bands. Insets in (a)-(g) and (i) display the electronic structure of the Dirac point at the Fermi energy, and sketches illustrate different channels for complex nearest-neighbor hopping [red (yellow) circles denote $p_{z}(s)$ orbitals, while $p_{x, y}$ orbitals are indicated by blue ellipsoids; black (red) arrows depicts $t_{\mathrm{NN}}$ hoppings (SOC hybridization), respectively]. Text below each panel denotes the hybridization of $\left\{p_{x}, p_{y}\right\}$ orbitals to $s$ and $p_{z}$ orbitals by kinetic hoppings, where $+(-)$ indicates (no) hybridization. For instance, "s $-\left\{p_{x}, p_{y}\right\}+p_{z}$ " in (a) and (i) means that there is no hybridization between $s$ and $\left\{p_{x}, p_{y}\right\}$, while $\left\{p_{x}, p_{y}\right\}$ and $p_{z}$ are coupled. 
TABLE I. Classification of the topological phases shown in Fig. 1 following Refs. 38 and 39, with corresponding topological invariant indicated in parentheses. Each entrance of the table corresponds to the respective situation presented in Fig. 1. Here, we have assumed that for the AII case the exchange field applied in (a), (b), (d), (e), (g), and (h) of Fig. 1 is set to zero, which results in a gapped TI phase. In the table, we list typical physical systems which realize mechanisms for generation of the TI $\left[\mathrm{AII}\left(\mathbb{Z}_{2}\right)\right]$ and QAHE $[\mathrm{A}(\mathbb{Z})]$ phases presented in Fig. 1.

\begin{tabular}{|c|c|c|c|}
\hline & \multicolumn{2}{|c|}{$\operatorname{AII}\left(\mathbb{Z}_{2}\right)$} & \multirow{2}{*}{$\begin{array}{l}\mathrm{A}(\mathbb{Z}) \\
\xi \mathbf{l} \cdot \mathbf{s}\end{array}$} \\
\hline & $\xi l_{z} s_{z}$ & $\frac{\xi}{2}\left(l^{+} s^{-}+l^{-} s^{+}\right)$ & \\
\hline$p_{z}$ & Silicene (Ref. 17) & Graphene (Ref. 4) & $\begin{array}{l}\text { Silicene in exchange } \\
\text { field (Ref. 21) }\end{array}$ \\
\hline$s$ & - & - & - \\
\hline$p_{x, y}$ & Spinful optical lattice (Ref. 36) & Bi(111) bilayers (Ref. 19) & $\begin{array}{l}\text { Bi(111) bilayers in exchange } \\
\text { field (Ref. 29) }\end{array}$ \\
\hline
\end{tabular}

where $\xi_{\text {flip }}=\xi\left(l^{+} s^{-}+l^{-} s^{+}\right) / 2$, and $t_{\mathrm{NN}}$ stands for the direct hybridization between $p_{x, y}$ orbitals on neighboring $\mathrm{A}$ and $\mathrm{B}$ sites. In analogy to the case with spin-conserving SOC considered previously, the effective hoppings within A and $\mathrm{B}$ sublattices are of opposite sign and the resulting gap is also topologically nontrivial. In this case, $t_{\mathrm{NNN}} \sim \xi^{2}$, and the corresponding gap $\Delta_{2}$ at $\mathrm{K}\left(\mathrm{K}^{\prime}\right)$ [Fig. 1(b)] scales quadratically with respect to the SOC strength. As in the previous case, the coupling between the spin-up and -down $p_{z}$ bands is forbidden, as seen in Fig. 1(b). In this figure, a small exchange field has been applied as well, and the corresponding Chern numbers of up and down bands are exactly the same as those for the case with the spin-conserving SOC only [Fig. 1(a)]. Therefore, without an exchange field, Fig. 1(b) corresponds exactly to the topological insulator phase in graphene, ${ }^{4}$ while the case of Fig. 1(a) describes the main origin of the topological insulator phase in silicene with buckled honeycomb lattice. ${ }^{17}$ Following the symmetry arguments, ${ }^{38,39}$ both cases are in the AII phase characterized by the $\mathbb{Z}_{2}$ invariant, as summarized in Table I.

Note that, while the arising nonzero Chern numbers of the bands in all situations discussed in Fig. 1 could lead to a QAH effect upon inducing a very large (larger than the bandwidth) exchange splitting in the system, we do not discuss such "trivial" possibility here. Thus, to induce nontrivial QAHE in the $p_{z}$ bands, the spin-up and -down $p_{z}$ bands have to be coupled, inducing exchange of the Chern numbers. This is not the case in two previous situations, in which no hybridization between the bands of different spin occurs at the Fermi energy, when an external exchange field is applied in the system [see Figs. 1(a) and 1(b)]. To achieve the opening of the gap at the Fermi energy due to the coupling of spin-up and -down states in this case, we have to allow for the hybridization between $p_{z}$ and $\left\{p_{x}, p_{y}\right\}$ orbitals upon taking the complete SOC into consideration, which results in opening of the gap due to spin mixing, and consequent exchange of the spin-up and -down Chern numbers [see Fig. 1(c)]. A possible transition process for $p_{z}$ orbitals realizing this scenario could read, for example, as follows:

$$
\left|p_{z}^{\mathrm{A} \uparrow}\right\rangle \stackrel{\xi_{\text {fiip }}}{\rightarrow}\left|p_{x, y}^{\mathrm{A}} \downarrow\right\rangle \stackrel{\xi_{l_{s} s_{z}}}{\rightarrow}\left|p_{z}^{\mathrm{A}} \downarrow\right\rangle .
$$

The magnitude of the gap, opened between up and down $p_{z}$ bands, as shown in Fig. 1(c), is proportional to $\xi^{2}$ according to our calculations. For the half-filled situation where the Fermi energy locates in the gap [inset of Fig. 1(c)], the anomalous Hall conductivity is proportional to the sum of the Chern numbers of all occupied bands, leading to nontrivial QAH effect. According to the symmetry classification of Refs. 38 and 39, this Chern insulator state corresponds to the A phase, characterized with the $\mathbb{Z}$ number equal to the Chern number (Table I).

The variation of Chern numbers upon band touching is determined by the Berry indices at degenerate points in a generalized parameter space $(\mathbf{k}, \eta)$, which is defined $a^{33,34}$

$$
\operatorname{Indx}_{\text {Berry }}=\frac{1}{2 \pi} \int_{\mathbf{S}^{2}} d s \Omega \cdot \mathbf{n},
$$

with $\mathbf{S}^{2}$ as a two-dimensional surface enclosing the degeneracy point, $\mathbf{n}$ is the surface normal vector pointing outwards, $\Omega$ is the Berry curvature. ${ }^{34}$ In our case, $\eta$ can be the strength of the exchange fields or SOC. Since $\mathbf{S}^{2}$ can be chosen arbitrarily small, the magnitude of Indx $x_{\text {Berry }}$ can be obtained by examining the band dispersion at the degeneracy point. ${ }^{33,34}$ For instance, if the dispersion of the crossing bands is linear (quadratic), the variation of the Chern number is one (two). This saves us from evaluating the Chern number exchange explicitly, although the sign of Indx $x_{\text {Berry }}$ can not be determined from this simple argument. In the case of Fig. 1(c), the Chern number of the upper valence band is reduced by 2 , while the Chern number of the lower conduction band is increased by 2, as compared to the situation in Figs. 1(a) and 1(b), when the hybridization between the $p_{z}$ bands of opposite spins is introduced. The reason for this is that, although the dispersion of the bands at the point of degeneracy is obviously linear [Fig. 1(c)], there are two such points in the Brillouin zone ( $\mathrm{K}$ and $\left.\mathrm{K}^{\prime}\right)$.

In previous studies of the QAHE in the context of graphene, ${ }^{20-24}$ an extended Kane-Mele model ${ }^{4}$ was employed with an additional Rashba term. The effective Rashba spinorbit interaction originates from the combination of intrinsic SOC and potential gradient perpendicular to the honeycomb lattice plane which breaks the inversion symmetry, and can be created by, e.g., applying a finite electric field perpendicular to the honeycomb plane ${ }^{25}$ or imposing a finite curvature of the honeycomb sheet. ${ }^{35}$ In this case, when the time-reversal symmetry is broken via, e.g., nonzero magnetization due to 
adatoms, the QAHE can be induced. ${ }^{9,10}$ Nevertheless, the effective complex hoppings due to the Rashba spin-orbit arise between $p_{z}$ orbitals of opposite spin on the nearest-neighbor sites, a situation topologically distinct from the complex NNN hoppings considered in this work.

\section{B. $s$ orbitals}

The second-order perturbation processes discussed above can also take place if the $s$ bands are taken instead of $p_{z}$ states. It is achieved by hybridization between $s$ and $p$ orbitals [Figs. 1(d)-1(f) and Table I]. For instance, the effective complex hoppings between $s$ orbitals can be induced by spin-conserving SOC via hybridizing with $\left\{p_{x}, p_{y}\right\}$ orbitals [Fig. 1(d)] or spin-flip SOC [Fig. 1(e)]. Comparing to the $p_{z}$ orbitals, the only difference is that for the case with spin-flip SOC [Fig. 1(e)], the corresponding virtual transitions are

$$
\left|s^{\mathrm{A} \uparrow}\right\rangle \stackrel{t_{\mathrm{NN}}}{\rightarrow}\left|p_{x, y}^{\mathrm{B}} \uparrow\right\rangle \stackrel{\xi_{\text {flip }}}{\rightarrow}\left|p_{z}^{\mathrm{B}} \downarrow\right\rangle \stackrel{\xi_{\text {flip }}}{\rightarrow}\left|p_{x, y}^{\mathrm{B}} \uparrow\right\rangle \stackrel{t_{\mathrm{NN}}}{\rightarrow}\left|s^{\mathrm{A} \uparrow}\right\rangle .
$$

Note that the role of $\left|p_{x, y}^{\mathrm{B}}\right\rangle$ and $\left|p_{z}^{\mathrm{B}}\right\rangle$ in this case can be exchanged, i.e., electrons can hop from $\left|s^{\mathrm{A}}\right\rangle$ to $\left|p_{z}^{\mathrm{B}}\right\rangle$ and then couple with $\left|p_{x, y}^{\mathrm{B}}\right\rangle$. Furthermore, similarly to the cases for $p_{z}$ bands [Figs. 1(a)-1(c)], the spin-up and -down $s$ bands are decoupled with Chern numbers of opposite sign [Figs. 1(d) and 1(e)], and a nontrivial QAH effect for the half-filled case takes place only when the two spin channels are entangled, i.e., due to hybridization with $p_{z}$ orbitals [Fig. 1(f)]. To the best of our knowledge, the realization of the nontrivial QAHE phase in $s$ bands has been not reported in the literature so far, and could serve as an interesting direction for materials research directed at engineering of Chern insulators.

\section{C. $\left\{p_{x}, p_{y}\right\}$ orbitals: Orbital purification}

More interestingly, nontrivial topological properties arise in the $\left\{p_{x}, p_{y}\right\}$ bands as well [Figs. 1(g)-1(i)]. If only the spinconserving SOC is considered, every $\left\{p_{x}, p_{y}\right\}$ band acquires a nonzero Chern number when the magnitude of SOC is larger than a critical value $\xi_{o p}$ [Fig. 1(g)]. This is different from the cases considered for $p_{z}$ and $s$ bands, where the strength of SOC determines only the size of the gap, while it is irrelevant for the topological properties of individual bands. Our analysis reveals that the origin of the nontrivial Chern numbers can be attributed to the so-called orbital purification, caused by strong SOC [Fig. 1(g)]. For instance, for the middle four bands in Fig. $1(\mathrm{~g})$, the Chern numbers are nonzero only when SOC is larger than $\xi_{o p}=5.4 \mathrm{eV}$, when the value of the orbital angular momentum $L_{z}$ in each of the bands becomes consistently either strictly positive or negative at each $k$ point [see the coloring of the bands in Fig. 1(g)]. This leads to the predominantly $m= \pm 1\left(p_{x} \pm i p_{y}\right)$ character of each band, which constitutes the essence of orbital purification. The complex NNN hoppings in this case are induced via the following mechanism:

$$
\left|p_{x}^{\mathrm{A}} \pm i p_{y}^{\mathrm{A}}\right\rangle \stackrel{V}{\rightarrow}\left|p_{x}^{\mathrm{B}} \mp i p_{y}^{\mathrm{B}}\right\rangle \stackrel{V}{\rightarrow}\left|p_{x}^{\mathrm{A}} \pm i p_{y}^{\mathrm{A}}\right\rangle,
$$

due to virtual transitions between the bands of different $m$ on different sublattices, mediated by kinetic hopping. Since only spin-conserving SOC is considered here, the spin-up and -down channels are not entangled [as clearly visible at the Fermi energy in Fig. 1(g)], if no direct hybridization of $\left\{p_{x}, p_{y}\right\}$ and other orbitals is present. In case the exchange field, applied in Fig. 1(g) for visibility of separate bands, was zero, the system would be in the QSH phase (see Table. I). This would correspond to the situation discussed by $\mathrm{Wu}^{36,37}$ for spinless honeycomb optical lattices.

On the other hand, for the lowest (topmost) two bands, the orbital angular momentum purification occurs for an even smaller SOC strength of about $0.3 \mathrm{eV}$, when only spin-conserving SOC is considered. There exists also another possibility for these bands to acquire a complex NNN hopping by spin-flip SOC as shown in Fig. 1(h). In this case, the Chern numbers for the middle four bands are always zero even if the spin-flip SOC strength is increased to very large values, while for the lowest (topmost) two bands, the Chern numbers are quantized with nonzero value. To obtain a nontrivial QAHE, entanglement between two spin channels is again essential, where Chern number exchange occurs via spin mixing as observed for the $s$ and $p_{z}$ cases above. For $\left\{p_{x}, p_{y}\right\}$ orbitals, such entanglement can be achieved by hybridization with $p_{z}$ orbitals, with corresponding Chern number exchange of 1 due to a linear band dispersion at the points of degeneracy. Following previous arguments, this leads to a nonzero QAH conductivity with $\mathcal{C}=-1$ at half-filling [Fig. 1(i)].

The orbital purification is determined by the competition between the strength of the onsite SOC and the bandwidth due to kinetic hoppings. In the model analysis above, we neglected the $\mathrm{V}_{\text {ррл }}$ hoppings in $\mathrm{Bi}$, with resulting $\xi_{\text {op }}$ of about $5.4 \mathrm{eV}$. This critical value is significantly reduced if $\mathrm{V}_{p p \pi}$ hoppings are taken into account, which reduces the bandwidth of the $p$ bands. In the latter case, for $\mathrm{Bi}, \xi_{o p}$ becomes $2.4 \mathrm{eV}$ if all relevant hopping integrals for the bilayers, as listed in Ref. 30, are included in our tight-binding calculations. Our first-principles calculations of the $\mathrm{Bi}(\mathrm{Sb})$ bilayer, on the other hand, show that the magnitude of the atomic SOC for $\mathrm{Bi}, \xi_{\mathrm{Bi}}$ ( $\xi_{\mathrm{Sb}}$ for $\left.\mathrm{Sb}\right)$, is about $2.8 \mathrm{eV}(0.9 \mathrm{eV})$. Thus, Bi bilayer resides in the strong SOC limit, where the orbital purification takes place, orbitally polarized $p_{x} \pm i p_{y}$ bands are formed and exhibit nonzero Chern numbers. To verify this explicitly, we evaluate the expectation value of the orbital momentum operator $L_{z}$ in Bi bands. As shown in Fig. 2, the first and the third valence bands of the $\mathrm{Bi}(111)$ bilayer are indeed orbitally purified in the entire Brillouin zone. The corresponding Chern numbers are \pm 2 and \pm 1 , respectively. The orbital purification in these bands occurs until the SOC strength of $\mathrm{Bi}$ atoms is scaled down to $70 \%$ of its atomic value, that is, approximately $2 \mathrm{eV}$, when the Bi bilayer loses its topological insulator properties [see Fig. 3(a)]. In this sense, we can call the Bi bilayer an orbital topological insulator, as opposed to the case of graphene, where all the topological properties are due to $p_{z}$ states. That is, due to strong atomic SOC strength of $\mathrm{Bi}$, orbital degrees of freedom can also play a role in inducing nontrivial topological phases (both TI and QAH insulator phases, Table I).

A comment on achieving nontrivial topological phases in optical lattices is in order, given that the idea of orbital angular moment purification was first proposed in the context of ultracold atoms. ${ }^{36}$ Quite recently, both Abelian ${ }^{40}$ and non-Abelian ${ }^{41-43}$ gauge fields (see Ref. 44 for a recent review) 


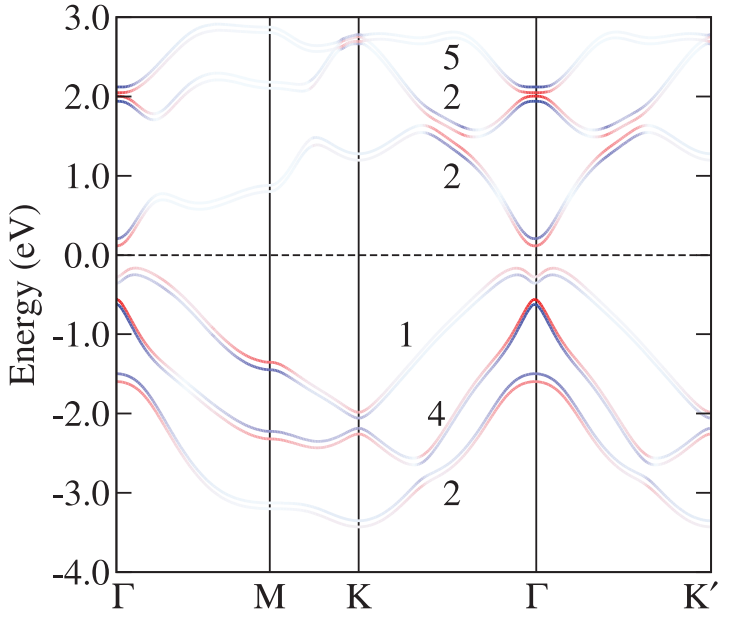

FIG. 2. (Color online) Orbital angular momentum $L_{z}$ purification in $\mathrm{Bi}(111)$ bilayer from first-principles calculations. Red (blue) stands for the expectation value of the $L_{z}$ operator. A small artificial exchange field of $10 \mathrm{meV}$ was applied perturbatively to split originally degenerate bands. In each pair of resulting nearly degenerate bands the expectation value of $L_{z}, s_{z}$, and the Chern numbers are opposite to each other. Integers next to the bands stand for the absolute value of the Chern numbers.

have been experimentally realized for trapped neutral atoms. Since ultracold atoms are spinless particles, in this context the relevance of the models discussed here is limited to the cases shown in Figs. 1(a), 1(d), and 1(g) when neglecting the spin degree of freedom. Moreover, the hybridization between different orbitals [as in Figs. 1(a) and 1(d)] is not necessary since in optical lattices complex hoppings are carried by dressed states. Experimentally, ultracold atoms in higher orbitals such as $p$ bands have been recently achieved (see, e.g., Ref. 45), which makes them a promising candidate for realizing various topological properties.

To summarize, we demonstrated that both orbital and spin degrees of freedom can be utilized to induce nonzero Chern numbers in an individual band, while the nontrivial QAH effect is due to the entanglement of two spin channels accompanied by spin exchange of the Chern number. In real materials, all bands are in general coupled by hybridization and, hence, Chern number exchange is expected at every nonaccidental band crossing. In this sense, graphene is a peculiar material ${ }^{46}$ since the well-separated in energy $\sigma$ and $\pi$ bands are not coupled by kinetic hopping due to graphene's planar structure. Sublattice staggering as found in silicene ${ }^{17}$ is too small to induce significant variation of the electronic structure, thus the simplified four-band model considering only the $\pi$ bands is good enough to account for the topological phase transitions in graphene-related systems. ${ }^{20-23}$ However, for $\mathrm{Bi}(111)$ bilayer and its derivatives, all three $p$ orbitals reside in the same energy scale, the hybridization between them is strongly enhanced due to buckling, and the strength of SOC is orders of magnitude larger as compared to that of carbon or silicon atoms. Therefore, rich physics with competing orbital and spin degrees of freedom is expected in the latter case. In the next section, we will demonstrate that nontrivial QAH phases can be achieved in $\mathrm{Bi}(111)$ BL by applying an exchange field, where the variation of Chern numbers can be explained using the Chern number exchange scheme illustrated above.

\section{TOPOLOGICAL STATES OF Bi AND Sb BILAYERS}

In the previous section, we illustrated within a tight-binding model how nonzero Chern numbers can arise due to intrinsic SOC and how to induce the QAH effect via applying an exchange field. One problem remains unsolved, however, that is the problem of characterizing various topological phases consistently. For instance, TIs are characterized by the $\mathbb{Z}_{2}$ index ${ }^{47}$ and QAH insulators by the (first) Chern number, and it is still not completely clear how to properly characterize the 2D insulating topological phases which do not fit into these two classes, e.g., insulators with broken time-reversal symmetry which originate from TIs and have a zero Chern number. Another issue is how such "intermediate" phases can be probed and distinguished experimentally from topological and Chern insulators. To this end, we use the spin Chern number first introduced by Sheng et al. ${ }^{48}$ and later generalized to the cases with spin-flip SOC by Prodan..$^{28}$ Compared to the more universal approach of Ref. 49, the spin Chern number can be easily constructed. It is applicable to the situations with or without time-reversal symmetry, and robust when the spectra of $P s_{z} P$ are gapped in the $\mathrm{BZ}$, where $P$ is the projection operator onto the occupied states. In the following, we use $\mathcal{C}_{+}$ $\left(\mathcal{C}_{-}\right)$to denote the "Chern" number of the spin-"up" (-"down") projected occupied bands, and the spin Chern number is defined as $\mathcal{C}_{\mathrm{s}}=\frac{1}{2}\left(\mathcal{C}_{+}-\mathcal{C}_{-}\right)$(for more details, see Ref. 29). As discussed in Sec. II, when time-reversal symmetry is not broken, $\mathcal{C}_{+/-}$are the Chern numbers of spin-up/spin-down bands, respectively, as depicted in Figs. 1(a) and 1(b), 1(d) and $1(\mathrm{e})$, and $1(\mathrm{~g})$ and $1(\mathrm{~h})$, and $\mathcal{C}_{\mathrm{s}}$ is equivalent to the $\mathbb{Z}_{2}$ number (Table I). On the other hand, in the QAH phase, the value of $\mathcal{C}_{\mathrm{s}}$ equals to the Chern number. Therefore, the variation of $\mathcal{C}_{\mathrm{s}}$ upon introducing perturbations into the system reflects the evolution of the corresponding topological phases. In this sense, the spin Chern numbers provide a consistent characterization of different topological phases.

In this section we tackle the question of finding and characterizing possible topological phases of $\mathrm{Bi}$ and $\mathrm{Sb}$ bilayers from first principles, as the corresponding Hamiltonian of these systems is altered by scaling the SOC matrix elements and applying an external exchange field. Our $a b$ initio calculations were performed using the full-potential linearized augmented plane-wave method as implemented in the Jülich density functional theory code FLEUR. ${ }^{50}$ The Wannier functions technique was used on top of self-consistent first-principles calculations to derive an accurate tight-binding Hamiltonian of the system. ${ }^{51-53}$ Both $\mathrm{Bi}(111)$ and $\mathrm{Sb}(111)$ bilayers considered in this work have buckled honeycomb lattice structure. The relaxed bulk in-plane lattice constant and the distance between the two layers for $\mathrm{Sb}(111)$ constitute 4.30 and $1.55 \AA$, respectively, while for $\operatorname{Bi}(111)$ bilayer the corresponding values are 4.52 and $1.67 \AA$. The details of calculating the anomalous (spin) Hall conductivities are described in Ref. 29. Moreover, we find that the spectra of $s_{z}$ projected onto the occupied states are always globally gapped for the cases considered in the following, despite the fact that the strength of $\mathrm{SOC}$ in $\mathrm{Bi}, \xi_{\mathrm{Bi}}$, is very strong 

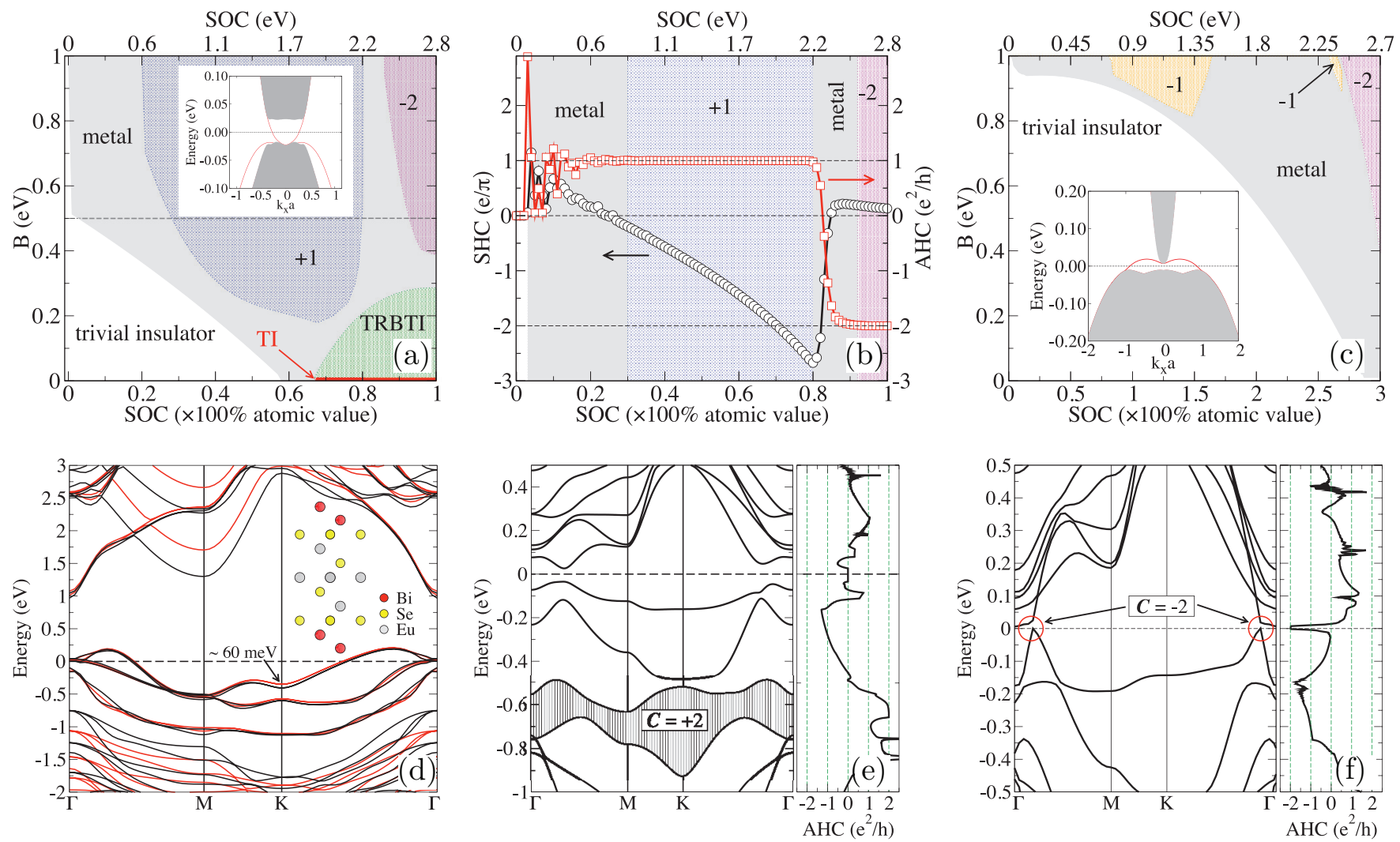

FIG. 3. (Color online) Quantum anomalous Hall phases in Bi/Sb(111) bilayers, and electronic structure of the Bi(111) bilayer on magnetic substrates and with magnetic dopants. (a) [(c)] displays the phase diagram of the $\mathrm{Bi}(111)$ [Sb(111)] bilayer with respect to the strength of atomic SOC and magnitude of exchange field $B$. Numbers denote the Chern number in the QAH phase, "TI" stands for the TI phase, while "TRBTI" stands for the time-reversal broken TI phase. The horizontal dashed line in (a) indicates the case with $B=0.5 \mathrm{eV}$, for which the AHC and spin Hall conductivity (SHC) are shown in (b) with respect to the strength of SOC. Inset in (a) displays the dispersion of the edge states (red lines) and the projected bulk states (gray shaded region) in $\mathrm{Bi}(111) \mathrm{BL}$ ribbon with $B=1 \mathrm{eV}$ and $70 \%$ of the Bi atomic SOC strength in the $\mathcal{C}=+1$ phase, while that in (c) displays the dispersion of the edge states in $\mathrm{Sb}(111) \mathrm{BL}$ ribbon with $B=1 \mathrm{eV}$ and $100 \%$ of the atomic $\mathrm{SOC}$ of $\mathrm{Sb}$ in $\mathcal{C}=-1$ phase. (d) displays the band structure of the $\mathrm{Bi}(111) \mathrm{BL}$ on top of EuSe(111) terminated with Se atoms. Black (red) lines denote the majority (minority) bands. The exchange splitting at the $\mathrm{K}$ point for the first two pairs of the valence bands is about $60 \mathrm{meV}$. The dashed horizontal line indicates $E_{F}$, and the inset displays the positions of the atoms. Left panel of (e) shows the electronic structures of $\mathrm{Bi}(111) \mathrm{BL}$ in $2 \times 2$ superstructure with one Fe atom located at the hollow site in-between the two Bi layers, whereas AHC conductivity is shown in the right panel. The horizontal dashed line in (d) indicates the position of the Fermi level which is not in the gap due to the fact that $\mathrm{Se}$ assimilate electrons. Shaded region in (e) marks the gap with the Chern number $\mathcal{C}=+2$. (f) is analogous to (e), but with the SOC strength of Bi scaled down to $20 \%$ of its atomic value.

$(\approx 2.8 \mathrm{eV})$, leading to well-defined spin Chern numbers. A uniform exchange field perpendicular to the honeycomb lattice plane was applied on top of the first-principles electronic structure, as described in Ref. 29. Note that for the buckled honeycomb lattice an in-plane exchange field can induce also nontrivial QAHE phases, ${ }^{54}$ where the distribution of magnetic flux in the hexagonal plaquette is different from the cases considered in this work. The spin-orbit strength $\xi$ in our calculations was scaled by hand via multiplying all atomic SOC matrix elements with a uniform scaling parameter during the self-consistency cycle. In order to clarify the effect of a more realistic exchange field, we have performed additional calculations of a $\mathrm{Bi}(111)$ bilayer on ferromagnetic substrates (europium chalcogenides) and in the presence of magnetic dopants in the system, as described later.

\section{A. Phase diagram of Bi(111) bilayer}

The calculated phase diagram of the Bi(111) BL with respect to the strength of atomic SOC $\xi$ and the magnitude of the exchange field $B$ is shown in Fig. 3(a). The emerging distinct topological phases can be characterized by the value of the AHC (which equals the Chern number times $e^{2} / h$ in the insulating regime) and the spin Chern number. The topological insulating phases are separated by a metallic phase, with topological phase transitions occurring during closing the bulk band gap and reopening it again as $B$ and the SOC strength are varied. As confirmed by the calculation of the spin Chern number, when the time-reversal symmetry is not broken $(B=0 \mathrm{eV})$, the $\mathrm{Bi}(111) \mathrm{BL}$ is a trivial insulator $\left(\mathcal{C}_{\mathrm{s}}=0\right.$, $\mathcal{C}=0$ ) for the SOC strength $\leqslant 56 \%$ of its atomic Bi value, while it turns into a $\mathrm{TI}\left(\mathcal{C}_{\mathrm{s}}=-1, \mathcal{C}=0\right)$ when $\xi$ is larger than 
$67 \%$ of $\xi_{\mathrm{Bi}} \cdot{ }^{19}$ In the TI phase, $\mathcal{C}_{+}=-\mathcal{C}_{-}=-1$, resulting in a spin Chern number of -1 .

Breaking the time-reversal symmetry by applying an exchange field induces topological phase transitions if the magnitude of the induced exchange splitting is large enough to make the two bands, which were originally below and above the gap, to overlap, according to the mechanism depicted in Figs. 1(c), 1(f), and 1(i). Before that, the original insulating phase is not destroyed, and to a certain extent preserves its topological properties in the presence of a small exchange field. For example, for $0.2 \xi_{\mathrm{Bi}}<\xi<0.56 \xi_{\mathrm{Bi}}$ the trivial insulator phase at $B=0$ retains as the $B$ is increased until the system enters the metallic phase in which the originally separated bands directly overlap. On the other hand, for $\mathrm{Bi} \mathrm{BL}$ with $\xi>0.67 \xi_{\mathrm{Bi}}$, the original topologically nontrivial insulating gap is finite for finite values of $B$, with $\mathcal{C}_{+}=-\mathcal{C}_{-}=-1$ until entering the metallic region. The resulting spin Chern number is in this case $\mathcal{C}_{\mathrm{s}}=-1$, which manifests the occurrence of the time-reversal broken TI (TRBTI) phase, observed also for graphene in Ref. 55, and discussed at length in, e.g., Ref. 29.

The transition into the QAH phase upon increasing the $B$ always takes place via an intermediate metallic phase [Fig. 3(a)]. In our previous work, we have shown the appearance of a $\mathrm{QAH}$ phase with $\mathcal{C}=-2$ for $\mathrm{Bi} \mathrm{BL}$ with full atomic SOC and $B \geqslant 0.42 \mathrm{eV}^{29}$ As we can see from Fig. 3(a), in a Bi BL with intermediate SOC strength, applying strong exchange field can also induce QAH phases which are derived from either metallic, trivial insulator, or topological insulating phases at $B=0$. For instance, in a Bi BL with SOC strength scaled to one half of the atomic value, a QAH phase with $\mathcal{C}=+1$ emerges for $B \geqslant 0.25 \mathrm{eV}$. It is an exotic phase, in which $\mathcal{C}_{-}=+1$ while $\mathcal{C}_{+}=0$, as compared to the QAH phase with $\mathcal{C}=-2$, in which $\mathcal{C}_{-}=\mathcal{C}_{+}=-1$. In the former case, the spin Chern number $\mathcal{C}_{s}=\frac{1}{2}\left(\mathcal{C}_{+}-\mathcal{C}_{-}\right)$is not an integer, and there exists only one chiral edge state localized at each edge of a one-dimensional $\mathrm{Bi}(111)$ ribbon, as shown in the inset of Fig. 3(a). Our calculations show that these edge states are spin polarized in the same direction, leading to the quantized value of the AHC (in units of $+e^{2} / h$ ) and also finite and large values of the SHC, as demonstrated explicitly by calculations for the case of $B=0.5 \mathrm{eV}$ in Fig. 3(b).

The peculiarity of the topologically nontrivial phases with nonzero $\mathcal{C}_{\mathrm{s}}$ lies in their finite $\mathrm{SHC}$. It can be understood in an intuitive way following two equations below:

$$
\begin{aligned}
& \mathrm{AHC}=\sigma^{\uparrow}+\sigma^{\downarrow}, \\
& \mathrm{SHC}=\sigma^{\uparrow}-\sigma^{\downarrow},
\end{aligned}
$$

where $\sigma^{\uparrow}\left(\sigma^{\downarrow}\right)$ denotes the conductivity of the majority (minority) electrons in units of $e^{2} / h(e / 4 \pi)$ for the AHC (SHC), respectively. These relations can be defined and hold true only when the spin-flip band transitions due to the spinnonconserving part of SOC are absent. In the trivial insulator phase, both the AHC and SHC are zero since $\mathcal{C}_{+}=\mathcal{C}_{-}=0$. When time-reversal symmetry is not broken, i.e., for the Bi BL in the TI phase, it implies that $\sigma^{\uparrow}=-\sigma^{\downarrow}=-1$, leading to zero AHC and to a quantized SHC of $-2 e / 4 \pi .{ }^{18}$ Nevertheless, due to the spin-flip part of SOC, the SHC of the Bi BL in the TI phase is reduced to about $0.7 e / 4 \pi$, as we found in our previous work (Fig. 3 in Ref. 29). In the QAH phase with $\mathcal{C}=-2$, $\mathcal{C}_{+}=\mathcal{C}_{-}=-1$, and, correspondingly, $\sigma^{\uparrow}=\sigma^{\downarrow}$, leading to zero SHC without the spin-flip transitions. However, the exchange splitting in the system leads to the fact that the spin-flip scattering between majority and minority states is not balanced, leading to a small but finite SHC, as shown in Fig. 3(b) for Bi BL at $B=0.5 \mathrm{eV}$.

On the contrary, for the QAH phase with the Chern number $\mathcal{C}=+1$, the majority spin channel is switched off since $\mathcal{C}_{+}=0$, and the resulting $\mathrm{SHC}$ is enhanced in magnitude as compared to the QAH phase with $\mathcal{C}=-2$ [see Fig. 3(b)]. As we artificially suppress the spin-flip band transitions by switching off the spin-nonconserving part of SOC in our calculations, we observe that the value of the SHC acquires a quantized value of the magnitude of $-\frac{e}{4 \pi}$ (not shown), despite the fact that the area of the $\mathcal{C}=+1$ phase shrinks as the electronic structure of the bilayer is modified when the spin-flip SOC is switched off. That is, we demonstrated that it is possible to achieve an exotic phase in which a large spin current is carried by topologically protected edge states. Since the number of edge states at each side of the ribbon equals to the Chern number $\mathcal{C}=1$ which prohibits scattering among the edge states on the same side, we suspect that the SHC is robust against spin-conserving perturbations. Since the value of the spin conductance in our case is comparable in magnitude to that of the QSH insulators, and it acquires a quantized value in the "spin-conserving" limit, we call such a topological phase a quantum spin Chern insulator phase.

The variation of the Chern numbers in the phase diagram of the Bi BL can be explained by the Chern number exchange mechanism at the critical points, as discussed in the previous section. ${ }^{33}$ For phase transitions at constant $\xi$ and varying $B$, the Chern numbers are changed by +1 (for intermediate $\xi$ ) and -2 (for large $\xi$ ) [Fig. 3(a)], corresponding to linear and quadratic dispersions at the critical points, respectively, as confirmed by our calculations (not shown). More interestingly, the Chern number is changed by -3 as we go from the $\mathcal{C}=+1$ phase to the $\mathcal{C}=-2$ phase at a constant large $B$ when varying the strength of SOC. Our analysis reveals that this transition can be decomposed into three steps, at each of which the topology of the Berry curvature distribution in the Brillouin zone changes, as analyzed in the following [Fig. $4(B=1 \mathrm{eV})]$.

We focus on the neighborhood of the $\Gamma$ point since most of the contribution to the variation of the Berry curvature and the Chern number exchange comes from the coupling of the highest occupied bands to the lowest unoccupied bands in this region. For $\xi \leqslant 0.79 \xi_{\mathrm{Bi}}$ in the $\mathcal{C}=+1$ phase, the distribution of Berry curvature exhibits a hot-loop structure with two pronounced singularitylike points at the $k_{x}=0$ axis. Enhancing the SOC strength brings down the conduction band and results in a singularity directly at the $\Gamma$ point as the bands touch each other. Upon reopening the gap at the touching $\Gamma$ point, the Chern number of the valence band is changed from +1 to +2 ( $\xi=0.80 \xi_{\mathrm{Bi}}$ in Fig. 4 ). If the valence band at this $\xi$ was separated by a global gap from the conduction band, the Chern number of the system would be +2 . As the $\xi$ is increased further, the conduction band goes further down in energy, while the point of band crossing with the valence band and corresponding singularities in the Berry curvature split 

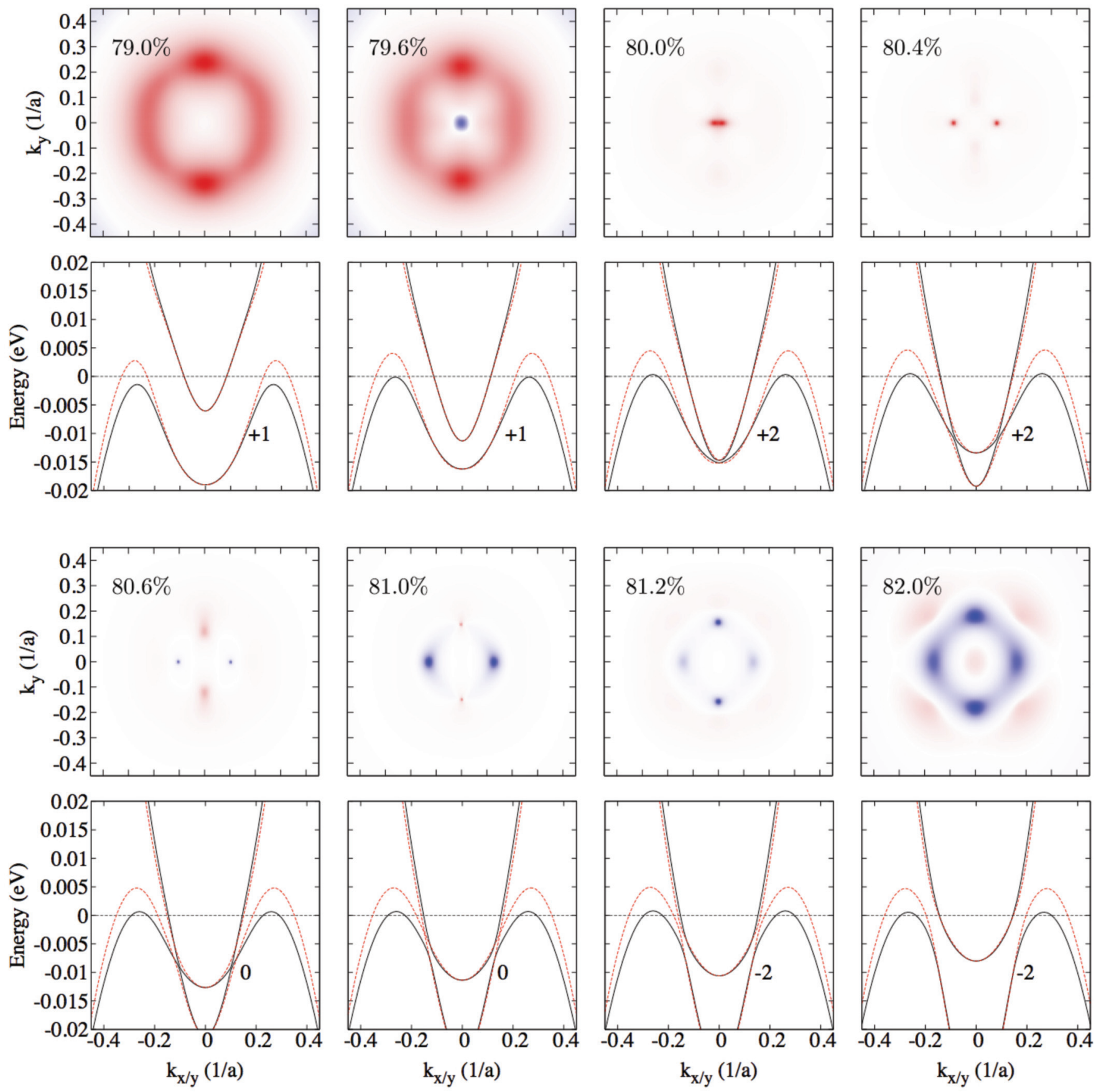

FIG. 4. (Color online) Variation of the distribution of the Berry curvature (first and third rows) and the band structure (second and fourth rows) at the $\Gamma$ point during a phase transition from $\mathcal{C}=+1$ to $\mathcal{C}=-2$ in $\mathrm{Bi}(111) \mathrm{BL}$ at $B=1 \mathrm{eV}$ as a function of the $\mathrm{SOC}$ strength. Numbers below each panel indicate the percentage of the strength of SOC in unit of $\xi_{\mathrm{Bi}}$. Black (red) lines stand for the bands with $k_{y}=0\left(k_{x}=0\right)$. The numbers stand for the Chern numbers of the topmost valence bands.

and move away from the $\Gamma$ point, resulting in a hot loop and four "monopoles" at $\xi=0.82 \xi_{\mathrm{Bi}}$. The Chern number of the valence band at each of such singular points is changed by -1 , therefore, the resulting QAH phase for $\xi \geqslant 82 \%$ of $\xi_{\mathrm{Bi}}$ has the Chern number $\mathcal{C}=-2$. At $82 \%$ of the Bi SOC strength, the band structure at the $\Gamma$ point is almost indistinguishable from that at $79 \%$ (see Fig. 4), although the Chern number, sign of the Berry curvature, and orbital character of valence and conduction bands have changed completely.

\section{B. Tuning $\xi$ in Bi bilayers: Alloying with $\mathrm{Sb}$}

We turn now to the question of how to achieve tuning of SOC strength and inducing a finite exchange field in a Bi(111) bilayer. Tuning the strength of SOC can be achieved by alloying $\mathrm{Bi}$ with its isoelectronic but lighter element $\mathrm{Sb}$, as demonstrated in, e.g., $\operatorname{BiTl}\left(\mathrm{S}_{1-\delta} \mathrm{Se}_{\delta}\right)_{2}$ where Se was substituted with the isoelectronic S. ${ }^{56}$ In Fig. 3(c), we show the calculated phase diagram of $\mathrm{Sb}$ (111) BL with respect to the magnitude of the exchange field $B$ and the strength of SOC $\xi$. The general 
features of the phase diagram of $\mathrm{Sb} \mathrm{BL}$ are similar to those of the Bi BL. For instance, at weak SOC strength and small magnetic field, both bilayers are trivial insulators, while QAH phases emerge with increasing $\xi$ and $B$. The QAH phase with Chern number $\mathcal{C}=-2$ emerges in $\mathrm{Sb} \mathrm{BL}$ for $B \geqslant 0.4 \mathrm{eV}$ if the SOC strength of $\mathrm{Sb}$ is scaled by about three times of its atomic value, to reach $\xi \approx 2.7 \mathrm{eV}$, which is very close to $\xi_{\mathrm{Bi}}$.

Clearly, there exist also significant differences between the phase diagrams in Figs. 3(a) and 3(c). First, the TI phase with time-reversal symmetry at $B=0$ is reached in Sb BL only when the SOC strength is scaled above $305 \%$ of its atomic value, $\xi \geqslant 2.75 \mathrm{eV}$, which is larger than the critical value in $\mathrm{Bi} \mathrm{BLs} \xi_{\mathrm{Bi}} \approx 1.9 \mathrm{eV}$. Second, the boundary between the trivial insulator and the metallic phases is moved towards larger $\xi$ and $B$ regime in $\mathrm{Sb} \mathrm{BL}$, as compared to $\mathrm{Bi} \mathrm{BL}$. Third, and most important, in the $\mathrm{Sb} \mathrm{BL}$ in the intermediate $\xi$ regime $\left(0.8 \xi_{\mathrm{Sb}} \leqslant\right.$ $\left.\xi \leqslant 1.8 \xi_{\mathrm{Sb}}\right)$ and in a larger exchange field, the QAH phase bears a Chern number with the sign opposite to that in the $\mathrm{Bi}$ $\mathrm{BL}: \mathcal{C}=-1$ for $\mathrm{Sb}$ as compared to $\mathcal{C}=+1$ for $\mathrm{Bi}$. We observe that the occurrence of the $\mathcal{C}=-1 \mathrm{QAH}$ phase is extremely sensitive to the fine details of the electronic structure of the bilayer. For instance, at $B=1 \mathrm{eV}$, a "-1" QAHE phase is also present in a small region of the SOC strength $2.6 \xi_{\mathrm{Sb}} \leqslant \xi \leqslant$ $2.65 \xi_{\mathrm{Sb}}$. Moreover, for $1.80 \xi_{\mathrm{Sb}} \leqslant \xi \leqslant 2.60 \xi_{\mathrm{Sb}}$ and $B \approx 1 \mathrm{eV}$, the $\mathrm{Sb}$ bilayer is gapped with a tiny gap of a few meV, which can not be measured in a realistic experiment in which disorder and temperature effects are inevitable.

The reason for the differences in the phase diagrams of $\mathrm{Sb}(111)$ and $\mathrm{Bi}(111) \mathrm{BLs}$ can be attributed to the difference in the fine details of their electronic structure. For instance, the magnitude of the $V_{p p \sigma}$ nearest-neighbor hopping parameter in $\mathrm{Bi}$ accounts to $80 \%$ of that in $\mathrm{Sb}$, while the value of the $V_{p p \sigma}$ next-nearest-neighbor hopping parameter in $\mathrm{Bi}$ is only half of that in $\mathrm{Sb}$, as listed in Ref. 30. This underlines that, to a certain extent, $\mathrm{Bi}_{1-x} \mathrm{Sb}_{x}$ alloys can not be treated within a simple SOC strength scaling picture, and we suspect that more interesting and nontrivial topological phases might occur in these alloys in a strong exchange field due to the competition between $\mathcal{C}= \pm 1$ phases, which have to be captured from more accurate first-principles calculations.

The QAH phase with $\mathcal{C}=-1$ found in Sb BL [Fig. 3(c)] is topologically different from the QAH phase with $\mathcal{C}=+1$ in Bi BL [Figs. 3(a) and 3(b)]. According to the bulk-edge correspondence, the bulk properties (Chern numbers) are reflected in the chirality of the edge states in a finite system. Obviously, the chiralities of the edge states for the $\mathcal{C}= \pm 1$ QAH phases are opposite to each other, as shown in the insets of Figs. 3(a) and 3(c). That is, the edge state located on the upper edge of a $\mathrm{Sb}(111)$ ribbon [inset of Fig. 3(c)] is right propagating, while that in a $\mathrm{Bi}(111)$ ribbon [inset of Fig. 3(a)] is left propagating. The SHC is of finite magnitude for both $\mathcal{C}= \pm 1$ phases [Fig. 3(b) for $\mathcal{C}=+1$ ], but of opposite sign due to different chiralities of the edge states. Overall, the \pm 1 QAHE phases in $\mathrm{Bi}$ and $\mathrm{Sb} \mathrm{BLs}$ are the quantum spin Chern insulator phases with opposite values of the anomalous and spin Hall conductivities.

\section{Tuning B in Bi bilayers: Magnetic substrates and doping}

In order to induce a finite exchange field in $\mathrm{Bi} / \mathrm{Sb}(111)$ bilayers, two realistic ways can be suggested: (i) either by depositing the bilayers on top of a suitable magnetic insulating substrate whose surface layer exhibits an in-plane ferromagnetic spin structure, or (ii) by doping the systems with magnetic atoms. We first briefly consider (i).

Europium chalcogenides $(\mathrm{Eu} X, X=\mathrm{O}, \mathrm{S}, \mathrm{Se}, \mathrm{Te})$ are magnetic semiconductors with diverse magnetic ordering and a wide range of lattice constants. ${ }^{57}$ We report here on firstprinciples calculations of $\mathrm{Bi}(111)$ bilayer on top of EuSe slabs along the (111) direction terminated with Se atoms [Fig. 3(d)], assuming ferromagnetic order in EuSe with magnetization direction perpendicular to the slab, but the conclusions below also hold for other europium chalcogenides. EuSe has cubic structure with the lattice constant of $6.19 \AA$, which provides the smallest lattice mismatch of around $3 \%$ to $\mathrm{Bi}(111) \mathrm{BL}$ as compared to other europium chalcogenides. To describe Eu $4 f$ electrons properly, we employed the LDA $+U$ scheme with $U=7.0 \mathrm{eV}$ and $J=1.2 \mathrm{eV}$. As shown in Fig. 3(d), the states of the system around $E_{F}$ are originated from Bi bands, with the largest exchange splitting induced by hybridization with the magnetic substrate accounting to about $60 \mathrm{meV}$. Such magnitude of the exchange splitting is definitely not enough to reach the QAH phase in Fig. 3(a), as verified by explicit AHC calculations (not shown), although it might be used to probe the time-reversal broken TI phase in the system, if the Fermi energy of the hybrid systems can be tuned to locate in the gap. The small exchange splitting in the BL is due to the hybridization with the half-filled shell of $4 f$ Eu electrons, which is quite localized leading to insignificant exchange interactions in the $6 p$ orbitals of $\mathrm{Bi}$ atoms. In this sense, using a substrate with partially filled $d$ shell, e.g., MnSe, ${ }^{58}$ might be favorable.

On the other hand, doping with or adsorption of $3 d, 4 d$, or $5 d$ transition-metal atoms can induce strong exchange splitting and hence a nontrivial QAH effect in TIs, as shown in Refs. 7-10. To demonstrate that this is indeed the case for the $\mathrm{Bi}$ bilayer, here we consider the case of Fe adatoms. Compared to graphene, the $\mathrm{Bi}(111)$ bilayer has a larger in-plane lattice constant (4.52 $\AA$, as compared to $2.46 \AA$ in graphene). Our structural relaxations show that the Fe atoms can be stabilized at the hollow site of the hexagonal plaquette in the middle of two $\mathrm{Bi}$ atomic layers at the spatial inversion point of the staggered honeycomb lattice. Figure 3(e) shows the electronic structure of Fe adatoms in $p(2 \times 2)$ superstructure on $\mathrm{Bi}(111)$ $\mathrm{BL}$, together with the calculated anomalous Hall conductivity. Among two global gaps which are formed upon Fe deposition, the band gap at the Fermi energy is trivial, while the band gap at about $0.6 \mathrm{eV}$ below $E_{F}$ exhibits a nonzero Chern number $\mathcal{C}=+2$ [Fig. 3(e)]. This is a new QAH phase, as compared to the phase diagram of Bi BL in Fig. 3(a), whose emergence is due to a strong hybridization between the $\mathrm{Fe} d$ and $\mathrm{Bi} p$ states in the vicinity of the Fermi energy, in analogy to the case of graphene. ${ }^{10,59}$ A similar mechanism also plays out for the $p(2 \times 2)$ Co doped $\mathrm{Bi}(111) \mathrm{BLs}$, in which case the QAH gap is opened at about $0.3 \mathrm{eV}$ below $E_{F}$ and the Chern number is +2 . Furthermore, varying the strength of SOC on $\mathrm{Bi}$ atomic sites can drive the system into yet different topological phases. For instance, as exemplified in Fig. 3(f), if we scale the strength of SOC of Bi atoms to $20 \%$ of their atomic value, the gap at $E_{F}$ of Fe-doped Bi BLs acquires nontrivial topological properties and displays a $\mathcal{C}=-2 \mathrm{QAH}$ phase. This manifests 
that the system of $3 d$ transition-metal adatoms/dopants on $\mathrm{BiSb}$ alloyed films definitely presents a unique and rich system in which exciting nontrivial phases could exist and could be tunable, suggesting thus the necessity for further theoretical and experimental investigations.

\section{SUMMARY}

In summary, using a generic tight-binding model constructed for buckled honeycomb lattice with $s p$ orbitals, we illustrated how to induce nontrivial topological phases by generating an effective complex next-nearest-neighbor hopping by spin-orbit interaction. Various channels can be recognized for $p_{z}, s$, and $\left\{p_{x}, p_{y}\right\}$ bands, respectively. We found that both spin and orbital degrees of freedom can be utilized to generate the complex hoppings, and verified that for a $\mathrm{Bi}(111)$ bilayer the orbital angular momentum purification plays an important role in its nontrivial properties due to the strong spin-orbit coupling strength of $\mathrm{Bi}$ atoms. Moreover, we showed how the nontrivial QAH phases can be induced by direct mixing of the two spin channels with corresponding exchange of the Chern number between the bands of opposite spin.

Further, we demonstrated that quantum anomalous Hall effect in the $\mathrm{Bi}(111)$ bilayer can be induced by a combined tuning of the strength of the spin-orbit interaction and the magnitude of an external exchange field. We observe that various topological phases of the $\mathrm{Bi}(111)$ bilayer, arising when the time-reversal symmetry is broken, can be characterized by
Chern and spin Chern numbers, corresponding to experimentally measurable transverse charge and spin conductivities. We showed that tuning the spin-orbit strength can be achieved by alloying $\mathrm{Bi}$ with $\mathrm{Sb}$, with rich physics expected in resulting $\mathrm{Bi}_{1-x} \mathrm{Sb}_{x}$ systems. In order to induce a finite exchange field in a $\mathrm{Bi}(111)$ bilayer, we examined the effect of a magnetic substrate, used for deposition of the bilayer, and magnetic dopants. We observe that $\mathrm{Bi}(111)$ bilayer on top of insulating europium chalcogenides exhibits a small exchange splitting of the bands, resulting in a time-reversal broken topological phase. On the other hand, we predict that a much larger splitting can be achieved upon introducing magnetic transition-metal impurities in the system, with resulting sizable QAH gaps in the spectrum of the composite system formed due to strong hybridization between the Bi $p$ and adatom's $d$ states. The position of these gaps and their topological properties can be further tuned by tuning the spin-orbit strength of the $\mathrm{Bi}(111)$ bilayer via, e.g., alloying with $\mathrm{Sb}$.

\section{ACKNOWLEDGMENTS}

We acknowledge insightful discussions with T. Schena and $\mathrm{C}$. Wu. This work was supported by the HGF-YIG Programmes VH-NG-513 and VH-NG-409, as well as by the DFG through Research Unit 912 and Grant No. HE3292/7-1. Computational resources were provided by the Jülich Supercomputing Centre. *h.zhang@fz-juelich.de

†y.mokrousov@fz-juelich.de

${ }^{1}$ N. Nagaosa, J. Sinova, S. Onoda, A. H. MacDonald, and N. P. Ong, Rev. Mod. Phys. 82, 1539 (2010).

${ }^{2}$ J. E. Hirsch, Phys. Rev. Lett. 83, 1834 (1999).

${ }^{3}$ Y. Kato, R. C. Myers, A. C. Gossard, and D. D. Awschalom, Science 306, 1910 (2004).

${ }^{4}$ C. L. Kane and E. J. Mele, Phys. Rev. Lett. 95, 226801 (2005).

${ }^{5}$ M. König, S. Wiedmann, C. Brüne, A. Roth, H. Buhmann, L. W. Molenkamp, X.-L. Qi, and S.-C. Zhang, Science 318, 766 (2007).

${ }^{6}$ F. D. M. Haldane, Phys. Rev. Lett. 61, 2015 (1988).

${ }^{7}$ C.-X. Liu, X.-L. Qi, X. Dai, Z. Fang, and S.-C. Zhang, Phys. Rev. Lett. 101, 146802 (2008).

${ }^{8}$ R. Yu, W. Zhang, H.-J. Zhang, S.-C. Zhang, X. Dai, and Z. Fang, Science 329, 61 (2010)

${ }^{9}$ Z. Qiao, S. A. Yang, W. Feng, W.-K. Tse, J. Ding, Y. Yao, J. Wang, and Q. Niu, Phys. Rev. B 82, 161414 (2010).

${ }^{10}$ H. Zhang, C. Lazo, S. Blügel, S. Heinze, and Y. Mokrousov, Phys. Rev. Lett. 108, 056802 (2012).

${ }^{11}$ H.-J. Zhang, X. Zhang, and S.-C. Zhang, arXiv:1108.4857.

${ }^{12}$ C.-Z. Chang, J.-S. Zhang, M.-H. Liu, Z.-C. Zhang, X. Feng, K. Li, L.-L. Wang, X. Chen, X. Dai, Z. Fang, X.-L. Qi, S.-C. Zhang, Y. Wang, K. He, X.-C. Ma, and Q.-K. Xue, Adv. Mater. 25, 1065 (2013).

${ }^{13}$ M. Z. Hasan and C. L. Kane, Rev. Mod. Phys. 82, 3045 (2010).

${ }^{14}$ X.-L. Qi and S.-C. Zhang, Rev. Mod. Phys. 83, 1057 (2011).

${ }^{15}$ B. Yan and S.-C. Zhang, Rep. Prog. Phys. 75, 096501 (2012).
${ }^{16}$ C. Liu, T. L. Hughes, X.-L. Qi, K. Wang, and S.-C. Zhang, Phys. Rev. Lett. 100, 236601 (2008).

${ }^{17}$ C.-C. Liu, W.-X. Feng, and Y. Yao, Phys. Rev. Lett. 107, 076802 (2011).

${ }^{18}$ S. Murakami, Phys. Rev. Lett. 97, 236805 (2006).

${ }^{19}$ M. Wada, S. Murakami, F. Freimuth, and G. Bihlmayer, Phys. Rev. B 83, 121310(R) (2011).

${ }^{20}$ W.-K. Tse, Z. Qiao, Y. Yao, A. H. MacDonald, and Q. Niu, Phys. Rev. B 83, 155447 (2011).

${ }^{21}$ T.-W. Chen, Z.-R. Xiao, D.-W. Chiou, and G.-Y. Guo, Phys. Rev. B 84, 165453 (2011).

${ }^{22}$ Z. Qiao, H. Jiang, X. Li, Y. Yao, and Q. Niu, Phys. Rev. B 85, 115439 (2012)

${ }^{23}$ M. Ezawa, Phys. Rev. Lett. 109, 055502 (2012).

${ }^{24}$ W. Beugeling, N. Goldman, and C. M. Smith, Phys. Rev. B 86, 075118 (2012).

${ }^{25}$ H. Min, J. E. Hill, N. A. Sinitsyn, B. R. Sahu, L. Kleinman, and A. H. MacDonald, Phys. Rev. B 74, 165310 (2006).

${ }^{26}$ C. Weeks, J. Hu, J. Alicea, M. Franz, and R. Wu, Phys. Rev. X 1, 021001 (2011).

${ }^{27}$ D. J. Thouless, M. Kohmoto, M. P. Nightingale, and M. den Nijs, Phys. Rev. Lett. 49, 405 (1982).

${ }^{28}$ E. Prodan, Phys. Rev. B 83, 195119 (2011).

${ }^{29}$ H. Zhang, F. Freimuth, G. Bihlmayer, S. Blügel, and Y. Mokrousov, Phys. Rev. B 86, 035104 (2012).

${ }^{30}$ Y. Liu and R. E. Allen, Phys. Rev. B 52, 1566 (1995). We neglected the parameters between the next-nearest neighbors in this paper, which are only relevant for the hoppings between bilayers. 
${ }^{31}$ C.-C. Liu, H. Jiang, and Y. Yao, Phys. Rev. B 84, 195430 (2011).

${ }^{32}$ S. Konschuh, M. Gmitra, and J. Fabian, Phys. Rev. B 82, 245412 (2010).

${ }^{33} \mathrm{~J}$. Bellissard, arXiv:cond-mat/9504030.

${ }^{34}$ M.-Y. Lee, M.-C. Chang, and T.-M. Hong, Phys. Rev. B 57, 11895 (1998).

${ }^{35}$ D. Huertas-Hernando, F. Guinea, and A. Brataas, Phys. Rev. B 74, 155426 (2006).

${ }^{36}$ C. Wu, Phys. Rev. Lett. 101, 186807 (2008).

${ }^{37}$ M. Zhang, H. H. Hung, C. Zhang, and C. Wu, Phys. Rev. A 83, 023615 (2011).

${ }^{38}$ A. P. Schnyder, S. Ryu, A. Furusaki, and A. W. W. Ludwig, Phys. Rev. B 78, 195125 (2008).

${ }^{39}$ A. Kitaev, AIP Conf. Proc. 1134, 22 (2008).

${ }^{40}$ Y. J. Lin, R. L. Compton, K. Jiménez-Garcia, J. V. Porto, and I. B. Spielman, Nature (London) 462, 628 (2009).

${ }^{41}$ Y. J. Lin, K. Jiménez-Garcia, and I. B. Spielman, Nature (London) 471, 83 (2011).

${ }^{42}$ P. Wang, Z. Q. Yu, Z. Fu, J. Miao, L. Huang, S. Chai, H. Zhai, and J. Zhang, Phys. Rev. Lett. 109, 095301 (2012).

${ }^{43}$ L. W. Cheuk, A. T. Sommer, Z. Hadzibabic, T. Yefsah, W. S. Bakr, and M. W. Zwierlein, Phys. Rev. Lett. 109, 095302 (2012).

${ }^{44}$ J. Dalibard, F. Gerbier, G. Juzeliunas, and P. Öhberg, Rev. Mod. Phys. 83, 1523 (2011).

${ }^{45}$ G. Wirth, M. Ölschläger, and A. Hemmerich, Nat. Phys. 7, 147 (2012).
${ }^{46}$ K. S. Novoselov, A. K. Geim, S. V. Morozov, D. Jiang, M. I. Katsnelson, I. V. Grigorieva, G. V. Dubonos, and A. A. Firsov, Nature (London) 438, 197 (2005).

${ }^{47}$ C. L. Kane and E. J. Mele, Phys. Rev. Lett. 95, 146802 (2005).

${ }^{48}$ D. N. Sheng, Z. Y. Weng, L. Sheng, and F. D. M. Haldane, Phys. Rev. Lett. 97, 036808 (2006).

${ }^{49}$ A. A. Soluyanov and D. Vanderbilt, Phys. Rev. B 85, 115415 (2012).

${ }^{50}$ For the description of the code, see www.flapw.de

${ }^{51}$ X. Wang, J. R. Yates, I. Souza, and D. Vanderbilt, Phys. Rev. B 74, 195118 (2006).

${ }^{52}$ F. Freimuth, Y. Mokrousov, D. Wortmann, S. Heinze, and S. Blügel, Phys. Rev. B 78, 035120 (2008).

${ }^{53}$ A. A. Mostofi, J. R. Yates, Y.-S. Lee, I. Souza, D. Vanderbilt, and N. Marzari, Comput. Phys. Commun. 178, 685 (2008).

${ }^{54}$ A. R. Wright, arXiv:1211.6519 (2012).

${ }^{55}$ Y. Yang, Z. Xu, L. Sheng, B. Wang, D. Y. Xing, and D. N. Sheng, Phys. Rev. Lett. 107, 066602 (2011).

${ }^{56}$ S.-Y. Xu，Y. Xia，L. A. Wray, S. Jia, F. Meier, J. H. Dil, J. Osterwalder, B. Slomski, A. Bansil, H. Lin, R. J. Cava, and M. Z. Hasan, Science 332, 560 (2011).

${ }^{57}$ See, for instance, N. M. Souza-Neto, D. Haskel, Y.-C. Tseng, and G. Lapertot, Phys. Rev. Lett. 102, 057206 (2009).

${ }^{58}$ W. Luo and X.-L. Qi, Phys. Rev. B 87, 085431 (2013).

${ }^{59}$ J. Hu, J. Alicea, R. Wu, and M. Franz, Phys. Rev. Lett. 109, 266801 (2012). 